\title{
Wolf-Rayet stars in OB associations
}

\author{
Andrzej Niedzielski and A. Strobel \\ Toruñ Centre for Astronomy, Nicolas Copernicus University, \\ ul. Gagarina 11, 87-100 Toruñ, Poland
}

\begin{abstract}
We present first results of our search for Wolf-Rayet members of $\mathrm{OB}$ associations, aiming in determination of distances and absolute magnitudes for WR stars.
\end{abstract}

\section{Introduction}

After the Hipparcos mission was completed, it became clear that the distances and the absolute magnitude scale of the Wolf-Rayet stars require re-thinking, in view of the considerable decrease of the distance to WR 11 (van der Hucht et al. 1997). However, Hipparcos data concerning paralaxes (distances) will not be of much help in this issue. Of all WR stars included in the Hipparcos program, only WR 11 has a parallax determination within $7 \sigma$ limit, another one within an, already unreliable, $3 \sigma$, five other stars within $2 \sigma$, followed by 18 within $1 \sigma$. A reliable Hipparcos distance hence has been obtained for only one WR star: $\gamma^{2}$ Velorum (WR 11)).

It seems obvious that one of possible approaches to the problem of distances and absolute magnitudes of WR stars may be based on the assumption of their large mass and young age. In other words, one may associate these objects with much better spatially localized young and massive relatives, like OB stars. Several recent studies of OB stars (Melnik\&Efremov 1995; Garmany\&Stencel 1992) suggest that at least some of these objects, present within OB associations, are spatially and physically coupled. Connecting WR stars with stellar groups and associations with known distances will allow to estimate their distances.

In this approach we try to apply the coincidence of coordinates of WR stars with those of OB associations, the kinematic information on WR stars and OB members (mainly from Hipparcos), and the photometric data available, as aid for association-membership assessment for WR stars.

\section{WR stars in OB associations}

In order to obtain a preliminary list of possible WR members of OB associations we combined the 7th Catalog of Galactic WR stars (van der Hucht 1999 and these Proceedings) with the new list of galactic OB associations of Melnik \& Efremov (1995 ME95). We found that positions of $33 \mathrm{WR}$ stars coincide with 10 association positions, assuming the association dimensions as given in ME95. Another group of $49 \mathrm{WR}$ stars coincides with associations if one doubles the association sizes. These numbers should be treated with great care, since many stars appear as possible members of several associations. Our preliminary list 
is therefore composed of $25 \mathrm{OB}$ associations. The existence of two of them (Sco 2A, Pis 20) is questioned in ME95. In most of them only one possible WR member appears but in some, like Cyg 1, 8, 9 or Car 2 as many as a dozen or more possible members were found. Altogether we found $82 \mathrm{WR}$ stars to be candidate $\mathrm{OB}$ association member.

As a next step, for every $\mathrm{OB}$ association containing WR candidates, we selected photometric and astrometric data from the Hipparcos catalog as well as from other available sources. First of all, we used the Hipparcos astrometric data $\left(\alpha, \delta, \mu_{\alpha}, \mu_{\delta}, \pi\right)$, which we transformed to the form $\left(\mathrm{l}, \mathrm{b}, \mu_{l}, \mu_{b}\right)$. Based on $\left(\mu_{l}, \mu_{b}\right)$ diagrams, we tried to find association members by distinguishing groups formed by the know members from the Galactic background. This step is possible only for WR stars with known astrometric data. Finally, photometric data were involved for both OB and WR stars.

\section{Results}

Keeping in mind the preliminary nature of our research (we have not completed yet the astrometric evaluation for all suspects) we can state that our procedure of selection of WR member candidates is more restrictive than that of Lundström \& Stenholm (1984) who found 41 WR stars to be members of 20 OB associations. Since we are rejecting candidates starting from the preliminary list, there can be no more candidates than selected by coordinates match. Therefore we can state that with respect to Lundström \& Stenholm (1984), several stars have lost their status as OB association members: WR 1 (Cas OB7), WR 2 (Cas OB1), WR 8 (Anon Pup a), WR 9 (Anon Pup b), WR 14 (Anon Vel a), WR 15 (Anon Vel b), WR 18 (Car OB1), WR 66 and WR 68 (Anon Cir), WR 74 and WR 75 (Nor OB4), WR 108 (Sgr OB1), WR 113 (Ser OB2), WR 114 and WR 115 (Ser OB1), WR 121 (Anon Sct), WR 127 (Vul OB2), WR 152, WR 153, WR 154 and WR 155 (Cep OB1).

The most promising 33 candidates $23(70 \%)$ have WN subtype, $9(27 \%)$ are WC stars, and one $(3 \%)$ is a WO. This curious preference of WN stars to coincide with the $\mathrm{OB}$ associations requires more attention. However, a more detailed discussion will have to wait for the completion of the association-member qualification with different criteria.

Acknowledgments. This paper was supported by the KBN grant 2P03D 00512 through N. Copernicus University.

\section{References}

Garmany, C.D., Stencel, R.E. 1992, A\&AS 94, 211

van der Hucht, K.A., Schrijver, H., Stenholm, B., Lundström, I., Moffat, A.F.J., Seggewiss, W., Setia Gunawan, D.Y.A., Sutantyo, W., van den Heuvel, E.P.J., De Cuyper, J.-P., Gómez, A., Marchenko, S.V. 1997, New Astronomy 2, 245

van der Hucht, K.A. 1999, in preparation

Lundström, I., Stenholm, B. 1984, A\&AS 58, 163

Melnik, A.M., Efremov, Y.N. 1995, PAZ 21, 13 